\title{
Reducción de emisiones de carbono por deforestación evitada en bosques del Espinal (Entre Ríos, Argentina)
}

\author{
Reduction of emissions by avoided deforestation in forests of Espinal \\ (Entre Ríos, Argentina)
}

\author{
Silvana María José Sione ${ }^{1 *}$. Hernán Jair Andrade ${ }^{2}$. Marcelo Germán Wilson ${ }^{3}$ \\ Leandro Javier Rosenberger $^{1 \oplus}$, María Carolina Sasal ${ }^{3}{ }^{\circledR}$, Silvia Gabriela Ledesma ${ }^{1}$ \\ y Emmanuel Adrián Gabioud ${ }^{3}$ (ำ
}

Sione, S. M. J., Andrade, H. J., Wilson, M. G., Rosenberger, L. J., Sasal, M. C., Ledesma, S. G. y Gabioud, E, A. (2021). Reducción de emisiones de carbono por deforestación evitada en bosques del Espinal (Entre Ríos, Argentina). Colombia Forestal, 24 (2), 39-51

Recepción: 17 de abril 2020

\section{Resumen}

Se estimó el carbono (C) almacenado en la biomasa aérea de bosques nativos del Espinal (Distrito del Ñandubay, provincia de Entre Ríos, Argentina) y la reducción de emisiones de $\mathrm{CO}_{2}$ por deforestación evitada. Se utilizó la información reportada en el Primer Inventario Nacional de Bosques Nativos del año 2007. Los bosques del área evaluada almacenaban a 2007 un total de $54.48 \mathrm{Tg}$ C, con un promedio de 38.82 Mg.ha-1 ${ }^{-1}$. En un escenario de deforestación cero a partir del año 2020 se lograría una reducción de emisiones del orden de 17.7 $\mathrm{Tg} \mathrm{CO}_{2}$ para el 2030, que representa un $3.7 \%$ de la meta nacional propuesta por Argentina de no superar, al año 2030, la emisión neta de $483 \mathrm{Tg} \mathrm{CO}$ (Contribución Nacionalmente Determinada). La conservación de los bosques del Espinal constituye, además, un mecanismo de gran potencial para generar y comercializar créditos de C según los estándares del mecanismo REDD+.

Palabras clave: cambio climático, cambio de uso de la tierra, carbono almacenado, conservación, ecosistemas boscosos.
Aprobación: 7 de abril 2021

\begin{abstract}
We estimated the carbon (C) stored in the total aerial biomass of native forests of the Espinal (Ñandubay District, Entre Ríos Province, Argentina) and the reduction of $\mathrm{CO}_{2}$ emissions by avoided deforestation. In this work, it was used the information reported in the First National Inventory of Native Forests from 2007. The forests from the evaluated area stored a total of $54.48 \mathrm{Tg} \mathrm{C}$, with an average

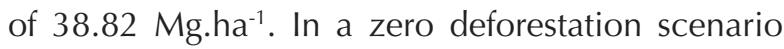
from 2020, an emission reduction of the order of 17.7 $\mathrm{Tg} \mathrm{CO}_{2}$ would be achieved by 2030, representing $3.7 \%$ of the national goal proposed by Argentina not to exceeding, the net emission of $483 \mathrm{Tg} \mathrm{CO}_{2}$ (Nationally Determined Contribution). The Espinal forests conservation also constitutes a mechanism with great potential to generate and commercialize C credits according to the standards of the REDD + mechanism.
\end{abstract}

Keywords: climate change, land use change, carbon stock, conservation, forest ecosystems.

\footnotetext{
Universidad Nacional de Entre Ríos (Argentina), Entre Ríos, Argentina. Universidad del Tolima (Colombia), Ibagué, Colombia. Instituto Nacional de Tecnología Agropecuaria, Argentina.

silvana.sione@fca.uner.edu.ar Autor para correspondencia.
} 


\section{INTRODUCCIÓN}

Los bosques desempeñan una función decisiva para determinar la acumulación de gases de efecto invernadero (GEI) en la atmósfera. Al actuar como sumideros de carbono $(\mathrm{C})$ absorben el equivalente a aproximadamente 2000 millones de toneladas de dióxido de carbono $\left(\mathrm{CO}_{2}\right)$ cada año (FAO, 2018). El IPCC (2007) estima que los bosques tienen un potencial de mitigación de 2.7-13.8 $\mathrm{Pg} \mathrm{CO}_{2}$.año-1 por su capacidad de capturar el $\mathrm{CO}_{2}$ atmosférico durante el proceso de fotosíntesis, lo que supone para el año 2050 un almacenamiento potencial de $100 \mathrm{Pg} \mathrm{C}$, que representa entre el $10 \%$ y el $20 \%$ de las emisiones de combustibles de origen fósil previstas para ese año. Frente a este escenario, la deforestación evitada, el manejo sostenible de los bosques nativos y la creación de nuevas áreas forestales representan importantes acciones a seguir.

El cambio de uso de la tierra constituye una de las principales causas del cambio global. En las últimas décadas, la degradación y conversión de los bosques a otro tipo de coberturas ha contribuido significativamente al aumento de las emisiones de GEI (IPCC, 2007). A fines de 2015, la $21^{\text {a }}$ Conferencia de las Partes (COP21) de la Convención Marco de Naciones Unidas sobre Cambio Climático (CMNUCC) consideró relevante el rol de la agricultura, la deforestación y los usos del suelo en las emisiones de GEI, responsables del $24 \%$ de las emisiones globales (IPCC, 2020).

La estrategia de reducción de emisiones debidas a la deforestación y la degradación de los bosques (REDD+), así como las funciones de la conservación, la gestión sostenible de los bosques y el incremento de las existencias forestales de C, resultarán cruciales para adoptar medidas a nivel mundial orientadas a combatir el cambio climático (FAO, 2018). La iniciativa REDD+, incluida en las agendas de la COP13 de la CMNUCC (UNFCCC 2007), busca además dar un vuelco al equilibrio económico hacia una gestión sostenible de los bosques para que sus valiosos bienes y servicios económicos, medioambientales y sociales puedan beneficiar a países, comunidades y usuarios de estos ecosistemas. A través de esta estrategia se busca que los bosques tengan un mayor valor en pie al que tendrían talados, puesto que se crea un valor financiero del C almacenado en los árboles (García-Idárraga, 2013). Newton y Benzeev (2018) y Pacheco et al. (2018) afirman que los compromisos de cero deforestación podrían apoyar la conservación de los bosques y la protección de los medios de vida rurales.

En Argentina, el sector forestal cumple un rol primordial para lograr la meta de no superar la emisión neta de $483 \mathrm{Tg} \mathrm{CO}_{2}$ al año 2030; meta establecida en la Contribución Determinada a Nivel Nacional (NDC por sus siglas en inglés) en el marco del cumplimiento de los objetivos del Acuerdo de París. Según el último Inventario Nacional de GEI (2017), la deforestación contribuye con el $14.5 \%$ de las emisiones totales del país (Programa Nacional ONU-REDD, 2019). Se estima que desde 1935 el país ha perdido cerca del $70 \%$ de sus bosques a un ritmo de 230000 ha.año $^{-1}$ entre 1998 y 2002, tasas que resultan mayores al promedio mundial (UMSEF, 2007). En el extenso territorio argentino, las causas, tipos y tasas de deforestación o degradación difieren según la zona ecológica. Esta situación hace necesario el desarrollo de esquemas de intervención a ser abordados por la estrategia REDD+ y que tengan en cuenta las condiciones específicas de cada ecorregión. En este contexto, se hace necesario contar con datos sólidos, consistentes y específicos sobre la cobertura boscosa, las emisiones y captura de $\mathrm{C}$ de las cubiertas forestales, y las dinámicas de deforestación, que deben ser medidos, monitoreados y reportados regularmente (Programa ONU-REDD, 2019).

El proceso de pérdida y degradación de los bosques nativos de Argentina condujo al gobierno nacional a regular su uso en función de su valor de conservación a través de la Ley N²6331/07 de Presupuestos Mínimos de Protección Ambiental de los Bosques Nativos. La provincia de Entre Ríos adhiere a esta legislación a través de la Ley $\mathrm{N}^{\circ}$ 10284/14, mediante la cual ordena sus bosques, 
pertenecientes a la provincia fitogeográfica del Espinal. Los principales motores de la deforestación en esta región son los precios de los productos agrícolas, los cambios tecnológicos y el contexto político y social. Sabattini et al. (2016) han estimado, para una cuenca representativa del Espinal (Entre Ríos), tasas anuales de deforestación del orden de $1.07 \%$ en el período 2001-2011.

Es prioritario cuantificar el C almacenado en los bosques del Espinal a fin de contribuir en la definición de estrategias de conservación que garanticen el mantenimiento de las reservas actuales y, con ello, la provisión de sus servicios ambientales. En este contexto, el objetivo de este trabajo fue estimar el $\mathrm{C}$ almacenado en la biomasa aérea de los bosques nativos del Distrito del Ñandubay, provincia fitogeográfica del Espinal (Entre Ríos, Argentina) y la reducción de emisiones potenciales de $\mathrm{CO}_{2}$ por deforestación evitada. Esta información aportará herramientas clave para estimar los créditos de $\mathrm{C}$ potenciales en futuros proyectos REDD+ de la región.

\section{MATERIALES Y MÉTODOS}

\section{Área de estudio}

El trabajo se realizó en el área de bosques nativos de la provincia fitogeográfica del Espinal (Entre Ríos, Argentina). Estos ecosistemas boscosos han sido tradicionalmente utilizados para la producción ganadera de cría bovina, constituyendo la base de los establecimientos agropecuarios. La vegetación presenta síntomas de alteración respecto a la vegetación prístina, propios del uso al que han sido destinados, derivados de los intensos procesos de colonización y de extracción de recursos naturales en la región.

El Espinal abarca $329395 \mathrm{~km}^{2}$ y se divide en tres distritos, según sus formaciones vegetales: del Ñandubay, del Caldén y del Algarrobo (Cabrera, 1976). El Distrito del Ñandubay se extiende por el este y sur de Corrientes, y el noroeste y centro de Entre Ríos. La temperatura media anual varía entre $18.8^{\circ} \mathrm{C}$ y $20.1^{\circ} \mathrm{C}$ y la precipitación media anual oscila entre 1190 y 1270 mm. Los biomas característicos son los bosques nativos semixerófilos, caracterizados por un estrato arbóreo inferior a $6 \mathrm{~m}$ de altura. Prosopis affinis Sprengel (ñandubay) y Vachellia caven Mol. (espinillo) son las especies arbóreas dominantes (Sabattini et al. 2016). En Entre Ríos, como producto del avance de la frontera agrícola, el Distrito del Ñandubay ha experimentado una reducción en el área de bosques nativos. A principios del Siglo XX, Raña estimó 2500000 ha de bosques nativos en la provincia (Muñoz et. al. 2005), alcanzando en el año 2007 una superficie de 1404128 ha (Secretaría de Ambiente y Desarrollo Sustentable de la Nación, 2007).

\section{Estimación de la biomasa aérea y el carbono almacenado en el estrato arbóreo}

Se utilizó la información reportada en el Primer Inventario Nacional de Bosques Nativos de Argentina (Secretaría de Ambiente y Desarrollo Sustentable de la Nación, 2007), específicamente para el Espinal, Distrito del Ñandubay (Entre Ríos). En este inventario se define a los bosques de ñandubay (ecosistemas característicos del Distrito del Ñandubay) como aquellos bosques xerófilos de ñandubay ( $P$. affinis) o espinillo ( $V$. caven) donde dichas especies presentan un área basal superior a $2 \mathrm{~m}^{2}$.ha ${ }^{-1}$ y constituyen un estrato arbóreo superior de densidad variable, dando lugar a formaciones desde abiertas a cerradas. El área basal promedio es de $6.1 \mathrm{~m}^{2} \cdot \mathrm{ha}^{-1}$. Especies acompañantes pueden ser el algarrobo negro (Prosopis nigra Griseb. Hieron.), quebracho blanco (Aspidosperma quebracho-blanco Schltdl.) y otras especies xerófilas. Dentro de los Bosques de Ñandubay, en función de su fisonomía, el inventario distingue tipos fisonómicos: por un lado, los bosques de ñandubay-espinillo (predominio de $P$. affinis o de $V$. caven, especies que contribuyen en más del $85 \%$ del área basal total), y por el otro, los bosques de 
ñandubay-espinillo con otras especies (bosques de mayor densidad arbórea, con un estrato arbóreo constituido por $P$. affinis o $V$. caven, que contribuyen en menos del $85 \%$ del área basal total, siendo frecuente que estas especies arbóreas xerófilas se entremezclen con otras más hidrófilas). A los fines del presente trabajo se consideró además la categoría bosques de ñandubay tipo parque, reportada como "Otras tierras forestales" en el inventario, referida a formaciones dominadas por $P$. affinis y $V$. caven en un estrato herbáceo continuo, con valores medios de área basal de $2 \mathrm{~m}^{2}$. ha ${ }^{-1}$ (Secretaría de Ambiente y Desarrollo Sustentable de la Nación, 2007).

La información utilizada para la estimación de la biomasa aérea y el carbono almacenado la constituyeron los datos de volumen por especie y total $\left(\mathrm{m}^{3} . \mathrm{ha}^{-1}\right)$ reportados en el inventario nacional para cada uno de los tipos fisonómicos descriptos, en la provincia de Entre Ríos (Tabla 1). Esta información ha sido generada a partir de muestreos de campo, con una intensidad de 23 unidades de muestreo (UM) para el Distrito del Ñandubay. Cada UM presentaba una superficie total de $1500 \mathrm{~m}^{2}$, conformada por un conglomerado de tres parcelas circulares de $500 \mathrm{~m}^{2}$. A su vez, cada una de estas parcelas estaba integrada por dos subparcelas concéntricas de forma circular: una de $500 \mathrm{~m}^{2}$, en la que se registraron todos los individuos leñosos con dap $\geq 10 \mathrm{~cm}$, y una subparcela de $12.5 \mathrm{~m}^{2}$, en la que se contaron y clasificaron por especie y tamaño todos los renovales con dap $<10 \mathrm{~cm}$.
La distribución de las UMs fue del tipo sistemática, sobre los vértices de una grilla de 18 x 18 km (Secretaría de Ambiente y Desarrollo Sustentable de la Nación, 2007).

Para cada tipo fisonómico se estimó la biomasa por especie y por hectárea a partir de la ecuación 1 :

$$
\mathrm{Bi}=\mathrm{Vi} * \mathrm{GEi} * \mathrm{FEBi} \quad \text { Ecuación } 1
$$

Donde:

Bi: biomasa aérea de la especie i $\left(\mathrm{kg} \mathrm{ha}^{-1}\right)$; Vi: volumen de la especie i $\left(\mathrm{m}^{3} \cdot \mathrm{ha}^{-1}\right)$; GEi: gravedad específica de la madera de la especie i $\left(\mathrm{kg} \cdot \mathrm{m}^{-3}\right)$; FEBi: factor de expansión de biomasa de la especie i.

Los valores de gravedad específica utilizados fueron $1050 \mathrm{~kg} \cdot \mathrm{m}^{-3}$ para $P$. affinis, $960 \mathrm{~kg} \cdot \mathrm{m}^{-3}$ para V. caven y $810 \mathrm{~kg} \cdot \mathrm{m}^{-3}$ para P. nigra (Atencia, 2003). Para otras especies fue utilizado el valor promedio de gravedad específica de las cinco especies acompañantes más frecuentemente presentes en estos bosques $\left(720 \mathrm{~kg} \cdot \mathrm{m}^{-3}\right)$. Se utilizaron valores de FEB de 3.7 para $P$. affinis (Sione et al., 2019a), 4.2 para V. caven (Sione et al., 2020a) y 4.9 para $P$. nigra (Sione et al., 2020b).

La biomasa arbórea aérea $\left(\mathrm{Mg} \cdot \mathrm{ha}^{-1}\right)$ fue calculada como la sumatoria de los valores de biomasa por especie para cada tipo fisonómico. La biomasa se convirtió a C aplicando el valor de 0.47 (Gasparri y Manghi, 2004; Sione et al., 2019b) y a $\mathrm{CO}_{2}$ con la constante estequiométrica de 3.67.

Tabla 1. Volumen por especie y total $\left(\mathrm{m}^{3} \cdot \mathrm{ha}^{-1}\right)$ para a cada tipo fisonómico de bosques nativos del Espinal (Distrito del Ñandubay, Entre Ríos, Argentina)

\begin{tabular}{lccccc}
\hline \multicolumn{1}{c}{ Tipo Fisonómico } & $\boldsymbol{P}$ affinis & V. caven & P. nigra & Otras especies & Total \\
\hline Bosques de Ñandubay-espinillo & 17.54 & 5.59 & - & 3.37 & 26.50 \\
Bosques de Ñandubay-espinillo con & 5.84 & 2.45 & 10.73 & 8.28 & 27.30 \\
otras especies & 4.38 & 2.10 & - & 0.62 & 7.10 \\
Bosques de Ñandubay tipo parque & &
\end{tabular}

Fuente: Secretaría de Ambiente y Desarrollo Sustentable de la Nación (2007, pp. 166-168). 
El C almacenado en el estrato arbóreo fue extrapolado a toda el área considerando la superficie del Distrito del Ñandubay (Entre Ríos), ocupada por cada tipo fisonómico: Bosques de Ñandubay-espinillo (575 753 ha), Bosques de Ñandubay-espinillo con otras especies (615 639 ha) y Bosques de Ñandubay tipo parque (212 736 ha). Finalmente, se calculó el valor medio ponderado de $\mathrm{C}$ almacenado $\left(\mathrm{Mg} \cdot \mathrm{ha}^{-1}\right)$ en función de la participación relativa de cada tipo fisonómico en el área total del Distrito del Ñandubay.

\section{Estudio de caso. Ensayo en Aldea Santa María}

Además de la información obtenida a partir de los datos del inventario nacional, se efectuó un estudio de caso en la localidad de Aldea Santa María (31 $37^{\circ} 34^{\prime \prime}$ latitud S, 6000`10" longitud O), perteneciente al Espinal, Distrito del Ñandubay. Esta localidad es un área de conservación obligatoria de suelos de la provincia de Entre Ríos, a la vez que constituye un Sitio Piloto del Proyecto GEF3623 (PNUD ARG/10/G49-PNUMA 4B85) de Incentivos para la Conservación de Servicios Ecosistémicos de importancia global en la Argentina. Resulta de particular interés la cuantificación del C almacenado en los bosques de este Sitio Piloto a fin de contar con herramientas para la valoración del servicio ecosistémico de captura y fijación de $\mathrm{CO}_{2}$ atmosférico.
La Aldea Santa María presenta una superficie total de 10040 ha, correspondiendo un 17\% (1706.8 ha) a tierras ocupadas actualmente por bosques nativos con un alto grado de fragmentación. Estos bosques han experimentado, al igual que el resto del Espinal, un régimen de disturbios intensos y continuos, tales como procesos de tala selectiva, deforestación y producción ganadera, sin un manejo forestal y ganadero planificado. Estas actividades han causado la reducción del área cubierta por bosques nativos y la degradación de los relictos de bosques. El 69\% de la superficie presenta suelos Vertisoles, de acuerdo al sistema de clasificación Soil Taxonomy (Wilson, 2007).

Allí fue desarrollado un ensayo de campo a escala de lote. En el año 2012 se realizó un muestreo aleatorio simple, con seis muestras localizadas en lotes ocupados por bosques nativos, los cuales se encontraban a una distancia promedio de $2 \mathrm{~km}$. Estos bosques resultan representativos del área evaluada respecto al estado de conservación e historia de uso, siendo destinados desde principios del siglo XX a la actividad ganadera de cría bovina. En cada uno de los lotes se estableció aleatoriamente una parcela de $1000 \mathrm{~m}^{2}(100 \times 10 \mathrm{~m})$ en las que se efectuaron mediciones en todos los individuos arbóreos de diámetro basal $\geq 10 \mathrm{~cm}$.

Tabla 2. Modelos de estimación de la biomasa arbórea y volumen aéreo utilizados para cada especie arbórea

\begin{tabular}{|c|c|c|c|c|}
\hline Especie & Modelo & $\mathbf{R}^{2}$ & Rango & Fuente \\
\hline Prosopis affinis "ñandubay" & $\operatorname{Ln}(B)=-3.23+2.61 * \operatorname{Ln}(d a b)$ & 0.97 & $5<\mathrm{dab}<35 \mathrm{~cm}$ & Sione et al. (2019a) \\
\hline $\begin{array}{l}\text { Prosopis nigra "algarrobo } \\
\text { negro" }\end{array}$ & $\mathrm{Va}=0.049 * \mathrm{dab}^{2.78}$ & 0.98 & $1<\mathrm{dab}<40 \mathrm{~cm}$ & Conti et. al. (2008) \\
\hline Vachellia caven "espinillo" & $\operatorname{Ln}(B)=-3.70+2.83 * \operatorname{Ln}(d a b)$ & 0.97 & $5<\mathrm{dab}<35 \mathrm{~cm}$ & Sione et al. (2020a) \\
\hline
\end{tabular}

Nota: Ln: logaritmo natural; B: biomasa aérea (kg.árbol-1); dab: Diámetro del tronco a la base (a $30 \mathrm{~cm}$ desde el suelo); Va: volumen aéreo $\left(\mathrm{dm}^{3}\right.$. árbol-1 $)$.

Fuente: Conti et al. (2018); Sione et al. (2019a; 2020a). 
Las variables medidas fueron: diámetro del tronco a la base -dab-, a $30 \mathrm{~cm}$ desde el nivel del suelo $-\mathrm{d}_{30^{-}}$, diámetro del tronco a la altura del pecho -dap- medidos con forcípula, altura de fuste medida desde la base del tronco hasta la primera bifurcación, y altura total con vara telescópica. En cada parcela se estimó la densidad absoluta y relativa por especie, calculada esta última como el porcentaje de individuos de cada especie arbórea sobre el total de individuos de la parcela.

La estimación de la biomasa aérea se realizó aplicando modelos alométricos de volumen $(P$. nigra) y de biomasa ( $P$. affinis y $V$. caven) desarrollados en la región del Espinal (Tabla 2).

Para individuos con diámetros basales fuera del rango de aplicación de los modelos alométricos, y para individuos de otras especies arbóreas, la biomasa se estimó a partir de la ecuación 2.

\section{$\mathrm{B}=\mathrm{Vf} * \mathrm{GE} * \mathrm{FEB} \quad$ Ecuación 2}

\section{Donde:}

B: biomasa aérea $\left(\mathrm{kg}\right.$.árbol $\left.{ }^{-1}\right)$; $\mathrm{Vf}$ : volumen de fuste $\left(\mathrm{m}^{3}\right.$.árbol $\left.{ }^{-1}\right)$; GE: gravedad específica de la madera $\left(\mathrm{kg} \cdot \mathrm{m}^{-3}\right)$; FEB: factor de expansión de biomasa.

Estas estimaciones permitieron cuantificar el peso seco de cada individuo arbóreo. Y a partir de la sumatoria de estos pesos individuales se determinó la biomasa seca total para cada parcela, que luego fue extrapolada a hectárea. Se convirtió la biomasa a $\mathrm{C}$, utilizando el procedimiento indicado anteriormente. Finalmente se calculó la biomasa y el C almacenado en el área total de bosques de la Aldea Santa María (1706.8 ha).

\section{Emisiones de $\mathrm{CO}_{2}$ evitadas}

El valor medio ponderado de C almacenado en los

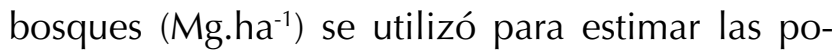
tenciales emisiones de $\mathrm{C}$, debido a la conversión de bosques nativos a otro tipo de cobertura (deforestación). Se plantearon dos escenarios para la estimación de estas emisiones potenciales de C (período 2020-2030): un escenario de referencia, donde se asume que la deforestación se mantiene en una tasa tasa anual de $1.07 \%$, valor estimado en un área representativa del Espinal (Sabattini et al., 2016), con la consiguiente reducción en el almacenamiento de $\mathrm{C}$ y aumento de las emisiones (ecuación 3); y un escenario con proyecto, en el que se asume que el $\mathrm{C}$ se mantiene en la biomasa aérea al evitarse la deforestación. Cabe destacar que este análisis no incluye los incrementos anuales de biomasa ni las emisiones por degradación forestal, y sólo se limita a estimar emisiones por deforestación.

$$
\mathrm{C}_{\mathrm{i}+1}=\mathrm{C}_{\mathrm{i}} * \mathrm{C}_{\mathrm{i}+1}=\mathrm{C}_{\mathrm{i}} *\left[\frac{100-D}{100}\right] \quad \text { Ecuación } 3
$$

Donde:

$\mathrm{C}_{i+1}$ : carbono almacenado en el año $\mathrm{i}+1(\mathrm{Gg}$ $\mathrm{CO}_{2}$ ) en el escenario de referencia, considerando el área boscosa total; $\mathrm{C} i$ : carbono almacenado en el año i $\left(\mathrm{Gg} \mathrm{CO}_{2}\right)$; D: tasa anual de deforestación (\%).

La diferencia entre $\mathrm{Ci}+t$ y $\mathrm{Ci}$ permite estimar la reducción anual del almacenamiento de $\mathrm{C}$ por efecto de la deforestación. Se utilizaron los valores medios de $\mathrm{C}$ estimados en el presente trabajo para el área total de bosques del Distrito del Ñandubay y para el área boscosa de la Aldea Santa María.

A partir de esta información se estimaron las emisiones de $\mathrm{CO}_{2}$ por deforestación (aplicando la constante estequiométrica de 3.67) con proyección para el período 2020-2030. Estas emisiones se estimaron considerando sólo la biomasa aérea. Cabe destacar que, en Argentina, el método de sustitución de bosques por tierras agrícolas consiste en el volteo de la vegetación, formación de escolleras y posterior quema, no contemplándose el uso de la madera. Aunque esta madera fuera utilizada, el destino predominante es para leña, por lo que también se la puede considerar pérdida como depósito de carbono. La emisión de $\mathrm{CO}_{2}$ se obtiene bajo el supuesto de que el $100 \%$ de la biomasa deforestada es transformada en $\mathrm{CO}_{2}$ por combustión y descomposición (Gasparri y Manghi, 2004). Las emisiones 
de $\mathrm{C}$ estimadas en este trabajo no contemplan el reemplazo de las áreas deforestadas por otra cobertura vegetal que capturen $\mathrm{CO}_{2}$ atmosférico, ni las diferentes tasas de descomposición de la biomasa removida.

Las estimaciones finales fueron transformadas a Tg y Gg (1Tg= 1000000 t; 1 Gg= 1000 t).

\section{RESULTADOS}

\section{Biomasa y almacenamiento de carbono}

Los valores de biomasa aérea variaron según el tipo fisonómico. Los bosques de Ñandubay-espinillo y los de Ñandubay-espinillo con otras especies presen-

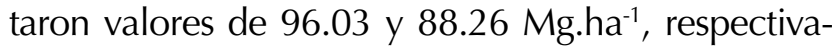
mente. Para los bosques de Nandubay tipo parque se estimaron valores significativamente inferiores (26.46 Mg.ha-1 ${ }^{-1}$. Considerando la superficie ocupada por cada tipo fisonómico se obtuvo un promedio ponderado de 82.10 Mg.ha-1 para el Distrito del Ñandubay (provincia de Entre Ríos). En la Tabla 3 se presenta la contribución de las especies arbóreas a la biomasa total por hectárea, para cada tipo fisonómico.

Las reservas de $\mathrm{C}$ en los bosques de Nandubay-espinillo y los de Ñandubay-espinillo con otras especies resultaron de 45.29 y $41.88{\mathrm{Mg} . \mathrm{ha}^{-1}}^{-1}$ respectivamente, en tanto que los Bosques tipo parque presentaron valores de $12.47 \mathrm{Mg} \mathrm{ha}^{-1}$. El promedio ponderado de C almacenado, obtenido a partir de las reservas de $\mathrm{C}$ y del área ocupada por cada tipo fisonómico, resultó de 38.82 Mg.ha-1. En los bosques de Ñandubay-espinillo y de Nandubay tipo parque la mayor contribución a las reservas de $C$ corresponde a $P$. affinis (70 y $64.4 \%$, respectivamente), en tanto que $P$. nigra constituye la especie de mayor aporte (47.7\%) en los bosques de Ñandubay-espinillo con otras especies (Tabla 3).

\section{Emisiones de CO2 evitadas}

Al año 2007, el distrito del Ñandubay presentaba una reserva total de $54.48 \mathrm{Tg} \mathrm{C}$ en sus bosques, lo que representa $199.9 \mathrm{Tg} \mathrm{CO}_{2}$ que fueron removidos de la atmósfera a lo largo de la existencia de estos ecosistemas. En el escenario con proyecto se asume que esta reserva de $\mathrm{C}$ se mantiene en el tiempo, dado que se conserva toda el área boscosa, evitando así emisiones de C a la atmósfera.

Las estimaciones efectuadas indican que en el período 2007-2019 las reservas de C en los bosques del Distrito del Ñandubay (provincia de Entre Ríos) se redujeron en $12.11 \%$ (6.6 Tg C), lo que equivale a emisiones del orden de 24.2 $\mathrm{Tg} \mathrm{CO}_{2}$ por deforestación (escenario de referencia). En este contexto, se espera que al 2030 las reservas de C sean de $42.5 \mathrm{Tg}$ C, que resultan $21.9 \%$ menor respecto al año 2007. Analizando el período 2020-2030, de mantenerse la tasa de $1.07 \%$ de pérdida anual de bosques nativos, la reducción de las reservas de $\mathrm{C}$ serían de $4.83 \mathrm{Tg}$ (0.48 Tg C.año ${ }^{-1}$ en promedio).

Tabla 3. Biomasa aérea total y por especie $\left(\mathrm{Mg}_{\mathrm{h}} \mathrm{ha}^{-1}\right)$ y carbono almacenado $\left(\mathrm{Mg} \cdot \mathrm{ha}^{-1}\right)$ para cada tipo fisonómico de bosques nativos del Espinal (Distrito del Ñandubay, Entre Ríos, Argentina)

\begin{tabular}{|c|c|c|c|c|c|c|}
\hline & \multicolumn{2}{|c|}{$\begin{array}{c}\text { Bosques de Ñandubay- } \\
\text { espinillo }\end{array}$} & \multicolumn{2}{|c|}{$\begin{array}{l}\text { Bosques de Nandubay- } \\
\text { espinillo con otras especies }\end{array}$} & \multicolumn{2}{|c|}{$\begin{array}{c}\text { Bosques de Ñandubay tipo } \\
\text { parque }\end{array}$} \\
\hline & $\begin{array}{l}\text { Biomasa } \\
\left(\text { Mg.ha }^{-1}\right)\end{array}$ & $\begin{array}{c}\text { C almacenado } \\
\left(\text { Mg.ha }^{-1}\right)\end{array}$ & $\begin{array}{l}\text { Biomasa } \\
\left(\text { Mg.ha }^{-1}\right)\end{array}$ & $\begin{array}{c}\mathrm{C} \text { almacenado } \\
\left({\left.\mathrm{Mg} . \mathrm{ha}^{-1}\right)}^{-1}\right.\end{array}$ & $\begin{array}{l}\text { Biomasa } \\
\left(\text { Mg.ha }^{-1}\right)\end{array}$ & $\begin{array}{c}\text { C almacenado } \\
\left(\mathrm{Mg}^{\prime} \mathrm{ha}^{-1}\right)\end{array}$ \\
\hline Prosopis affinis & 68.15 & 32.03 & 22.70 & 10.67 & 17.04 & 8.01 \\
\hline Vachellia caven & 22.54 & 10.60 & 9.86 & 4.64 & 8.44 & 3.97 \\
\hline Prosopis nigra & - & - & 42.58 & 20.01 & - & - \\
\hline Otras especies & 5.33 & 2.66 & 13.12 & 6.56 & 0.98 & 0.49 \\
\hline Total & 96.03 & 45.29 & 88.26 & 41.88 & 26.46 & 12.47 \\
\hline
\end{tabular}




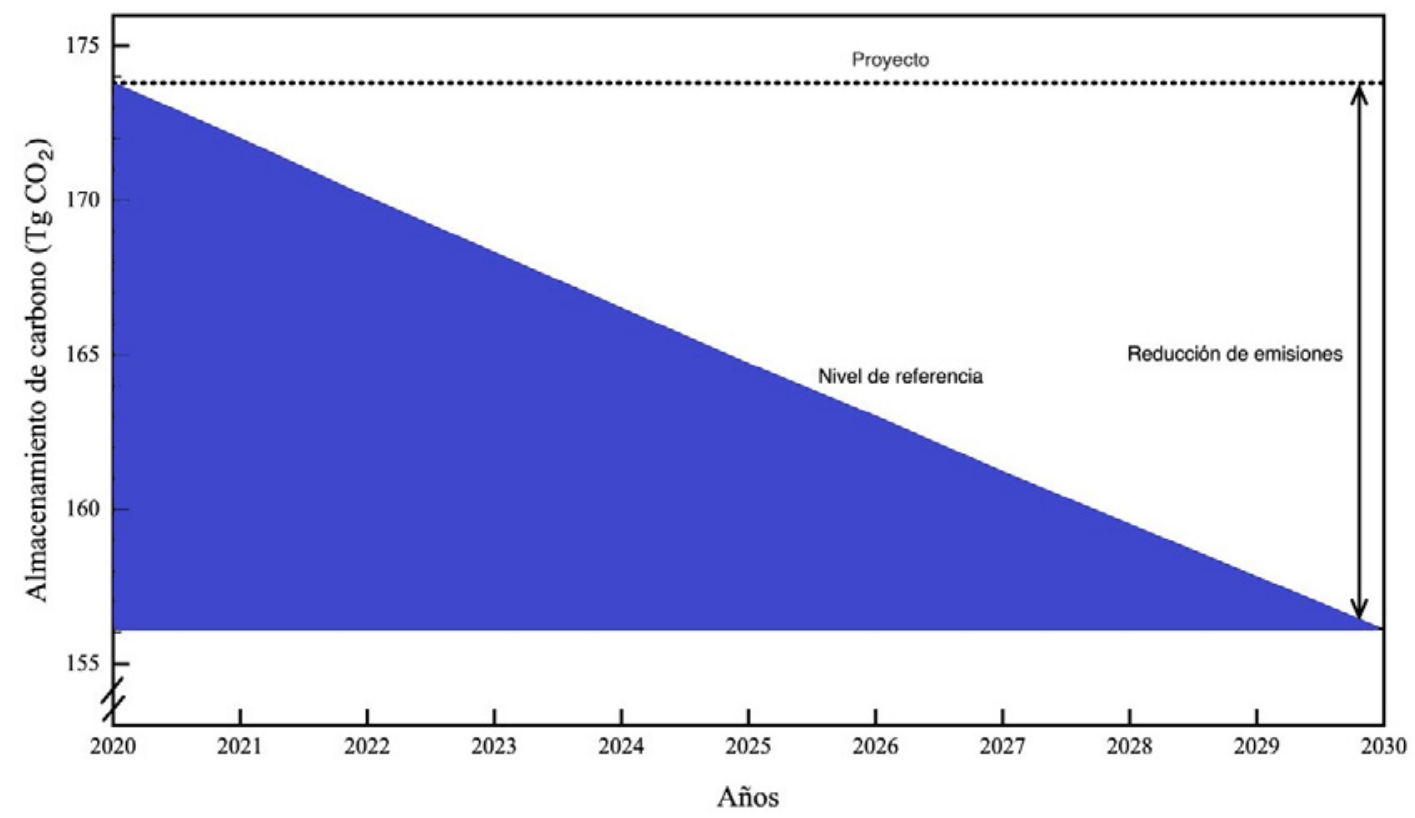

Figura 1. Proyección del almacenamiento de carbono en la biomasa aérea de bosques nativos del Espinal (Distrito del Ñandubay, Entre Ríos, Argentina) al año 2030, bajo las tasas de deforestación regionales $\left(1.07 \%\right.$ año $\left.{ }^{-1}\right)$. Las áreas marcadas indican emisiones de $\mathrm{CO}_{2}$ que podrían reducirse por deforestación evitada.

Las emisiones potenciales de $\mathrm{C}$ en este período debido a la deforestación serían de 17.7 $\mathrm{Tg} \mathrm{CO}_{2}$, que podrían evitarse si se detuviera la deforestación (Figura 1).

\section{Estudio de caso. Ensayo en Aldea Santa María}

Los bosques evaluados responden al tipo fisonómico de Bosques de Ñandubay-espinillo con otras especies, dado que la contribución conjunta de $P$. affinis y $V$. caven al área basal total resulta inferior al $85 \%$, oscilando entre $17.6 \%$ y $84 \%$ en las parcelas analizadas.

La densidad arbórea media total de los bosques evaluados fue de 528 individuos. ha ${ }^{-1}$, siendo $P$. affinis la especie con la mayor densidad relativa, seguida por $P$. nigra y $V$. caven $(59.3 ; 18.3$ y $12.9 \%$, respectivamente). Prosopis alba, C. ehrenbergiana y Geoffroea decorticans (Gill. ex Hook. \& Arn.) Burkart resultaron especies acompañantes que en conjunto representaron el $9.5 \%$ de la densidad total.

P. nigra presentó los individuos de mayores diámetros, con valores promedios de $30.0 \pm 5.6 \mathrm{~cm}$ de dab. En contraste, los menores valores para esta variable correspondieron a $V$. caven $(19.7 \pm 1.3 \mathrm{~cm})$. $P$. affinis presentó dab promedios de $22.7 \pm 4.4 \mathrm{~cm}$. Los individuos de $P$. nigra presentaron los mayores valores de altura total $(6.2 \pm 1.1 \mathrm{~m}$ en promedio) $P$. affinis y $V$. caven presentaron alturas menores $(3.9 \pm 0.7 \mathrm{~m}$ y $4.1 \pm 0.8 \mathrm{~m}$, respectivamente).

La estructura por clases diamétricas de los bosques analizados fue heterogénea. El $64 \%$ de los individuos de $P$. affinis (313 individuos.ha ${ }^{-1}$ ) presentaron un dab inferior a $25 \mathrm{~cm}$. La mayoría de los individuos de $V$. caven (85\%) registraron dab inferiores a $25 \mathrm{~cm}$, siendo la clase de $15-19.9 \mathrm{~cm}$ la más abundante (39\%). La estructura diamétrica resultó diferente en $P$. nigra, especie en la que más del $60 \%$ de los individuos inventariados tenían dab superiores o igual a $30 \mathrm{~cm}$, con un $27.6 \%$ de los individuos con dap $>45 \mathrm{~cm}$.

\section{Biomasa y almacenamiento de carbono}

La biomasa aérea fue de $84.30 \mathrm{Mg} \cdot \mathrm{ha}^{-1}$, resultando similar al valor obtenido a partir de la información 
del inventario nacional para el tipo fisonómico Bosques de Ñandubay-espinillo con otras especies $\left(88.26 \mathrm{Mg} \mathrm{ha}^{-1}\right)$. P. nigra y $P$. affinis contribuyeron con el $55 \%$ y $32 \%$, respectivamente, mientras que V. caven sólo contribuyó con el 7\%. La biomasa aportada por las especies acompañantes fue de

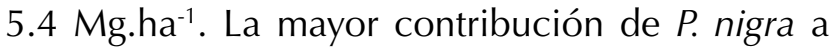
la biomasa total obedece a la mayor biomasa individual de esta especie, la que resultó significativamente mayor $(\mathrm{p}<0.05)$ respecto a las otras especies (Figura 2).

El C almacenado en la biomasa fue de 39,6 Mg.ha- ${ }^{-1}$, con una mayor contribución de P. nigra, la cual resultó estadísticamente similar $(\mathrm{p}>0.05)$ a $P$. affinis pero significativamente superior $(p<0.05)$ a $V$. caven (Figura 2). El C almacenado en la biomasa de especies acompañantes alcanzó 2.7 Mg.ha-1. Las especies de Prosopis y $V$. caven aportan el 93.3\% del total de $\mathrm{C}$ almacenado en la biomasa.

Considerando el área cubierta por bosques nativos (1706.8 ha), las reservas totales de $C$ en la biomasa aérea son del orden de $67.6 \mathrm{Gg}$, que representan $248.1 \mathrm{Gg} \mathrm{CO}_{2}$.

\section{Emisiones de CO2 evitadas}

En el escenario de referencia, donde se asume que se mantiene la deforestación a la tasa anual simulada $(1.07 \%)$, las reservas de $C$ al año 2030 se reducirían un $17.6 \%$ respecto a las estimaciones efectuadas para el año 2012, alcanzando 55.7 Gg. Analizando el período 2020-2030, la disminución en las reservas de C sería del orden de $6.3 \mathrm{Gg}$, por lo que se estima que en el escenario con proyecto se podría evitar un total de $23.2 \mathrm{Gg}$ de emisiones de $\mathrm{CO}_{2}$ a la atmósfera por deforestación evitada $\left(2.3 \mathrm{Gg} \mathrm{CO}_{2} \cdot \mathrm{año}^{-1}\right)$.

\section{DISCUSIÓN}

Winjum et al. (1993) afirman que la biomasa arbórea de bosques naturales de 12 países, que representan aproximadamente el $60 \%$ de los ecosistemas boscosos del planeta (incluyendo bosques boreales, templados y tropicales), almacena un total de 137.5 Pg C con una media de 47.4 Mg C.ha-1 y un rango muy variable $\left(15-126 \mathrm{Mg} \mathrm{C} \mathrm{ha}^{-1}\right)$.

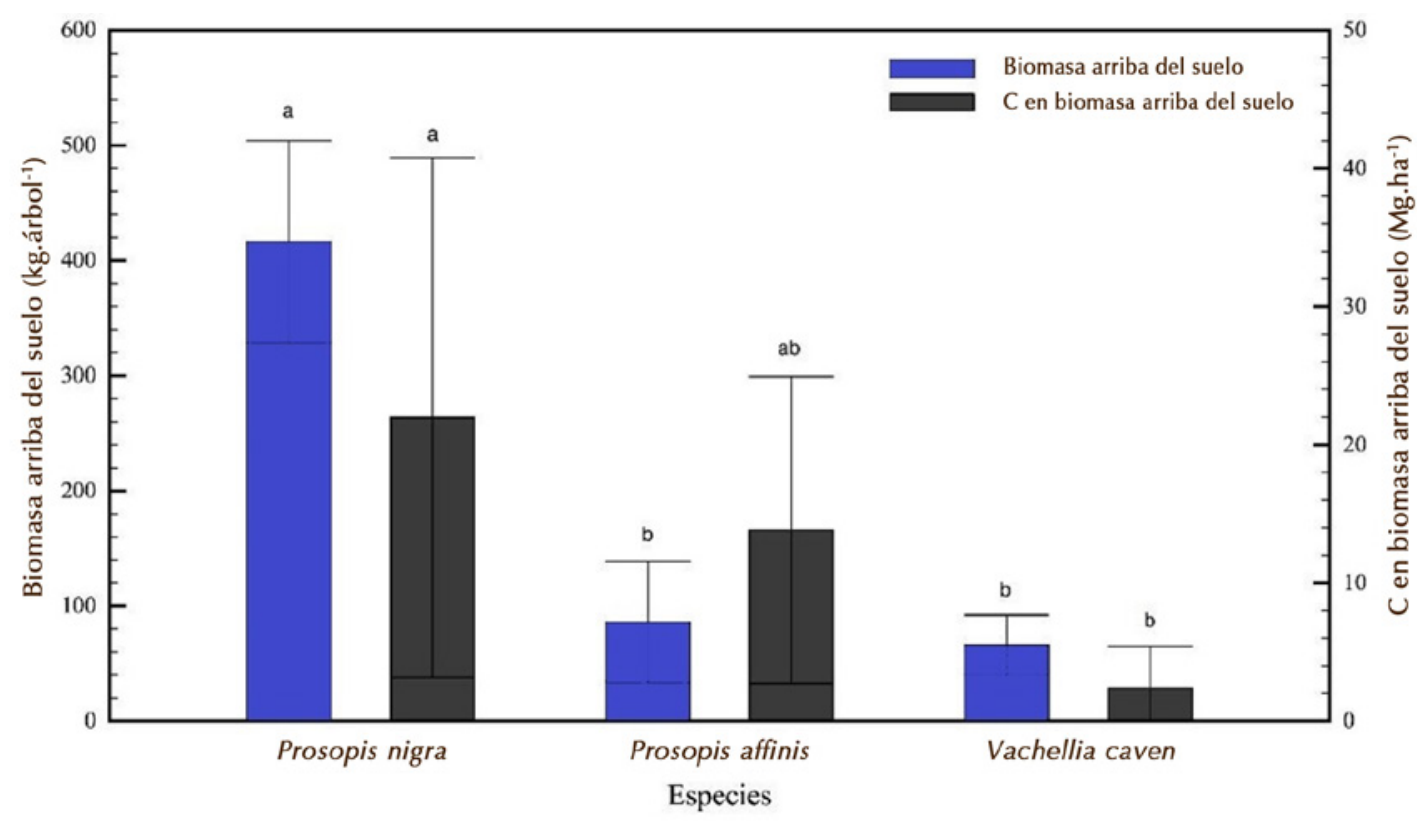

Figura 2. Biomasa aérea por especie y carbono almacenado en la biomasa arbórea por hectárea en bosques nativos de la Aldea Santa María (Espinal, Distrito del Ñandubay, Entre Ríos, Argentina). Letras diferentes indican diferencias significativas entre especies (Test LSD, $\mathrm{P}<0.05$ ). Las barras de error indican error estándar. 
En biomas forestales de distintas regiones del mundo, Pardos (2010) reportó valores dispares en el C almacenado y los agrupó en tres catego-

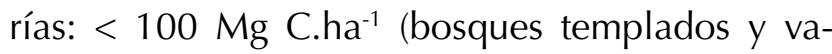
lores medios de bosques europeos); entre 100 y 250 Mg C.ha-1 (algunos bosques de coníferas, bosque tropical secundario y sistemas agroforestales) y $>300 \mathrm{Mg} \mathrm{C} \mathrm{ha}^{-1}$ (bosques tropicales y bosque de frondosas europeo). Los valores sensiblemente mayores de los bosques tropicales marcan una clara diferencia del resto. Este autor considera que la estructura de las masas arbóreas, su composición de especies, los aprovechamientos de madera y los agentes destructores bajo los que han estado sometidos los biomas podrían justificar las diferencias encontradas.

Los valores de biomasa aérea y de reservas de C estimados en este trabajo para bosques del Espinal, Distrito del Ñandubay (provincia de Entre Ríos), resultan inferiores a los obtenidos en otras regiones forestales argentinas. Gasparri y Manghi (2004) han estimado valores de biomasa aérea que osci-

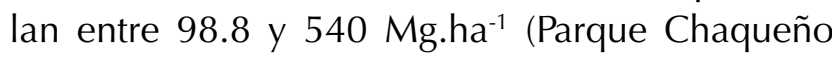
y Bosques andino-patagónicos, respectivamente), mientras que para las Selvas tucumano-boliviana y misionera estos valores resultan intermedios

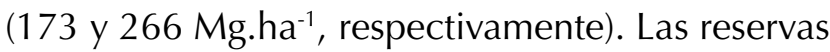
de $\mathrm{C}$ para estas regiones varían entre 49.4 Mg.ha-1

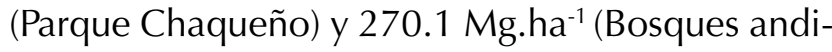
no-patagónicos). Por su parte, en bosques del Chaco Árido, Conti et al. (2014) estimaron que, en su estado más conservado, la biomasa vegetal aérea almacena 43.3 Mg C.ha-1 ${ }^{-1}$ El Parque Chaqueño resulta la región con valores de biomasa y reservas de $\mathrm{C}$ más próximos a los estimados en este trabajo. Esto obedece a su similitud con el Espinal, pues ambas regiones forestales presentan bosques xerófilos caducifolios con especies arbóreas en común.

Según las estimaciones obtenidas en el presente estudio, si en el período 2020-2030 la superficie cubierta por bosques nativos en el Espinal (Distrito del Ñandubay, provincia de Entre Ríos) se involucrara en la opción de deforestación evitada prevista en el marco del mecanismo REDD+, se reducirían emisiones del orden de $17.7 \mathrm{Tg} \mathrm{CO}_{2}$ (1.77 Tg.año $\left.{ }^{-1}\right)$. La reducción de emisiones por deforestación evitada en esta región representa un $3.7 \%$ de la meta propuesta por Argentina de no superar, al año 2030, la emisión neta de 483 Mt $\mathrm{CO}_{2}$ (483 $\mathrm{Tg} \mathrm{CO} \mathrm{CO}_{2}$ ), establecida en su Contribución $\mathrm{Na}$ cional Planeada del año 2016.

En la provincia del Chaco se han estimado valores de emisiones de $\mathrm{CO}_{2}$ por deforestación. A partir de la pérdida de tierras forestales en el año 2014 (18.563 ha) se estimaron emisiones anuales de 2.97 $\mathrm{Tg} \mathrm{CO}_{2}$ (MAyDS, 2017). En Argentina, en el marco de la etapa de preparación del mecanismo REDD+ se han estimado emisiones brutas de $\mathrm{CO}_{2}$ por deforestación para cada una de las regiones forestales del país, a fin de estimar el $\mathrm{Ni}$ vel de Referencia de Emisiones Forestales (NREF). EI NREF fue calculado como el promedio histórico de emisiones de $\mathrm{CO}_{2}$ por deforestación para el período histórico 2002-2013. En total se emitieron 1.214 Mt $\mathrm{CO}_{2}$ en todo el período histórico. La región del Parque Chaqueño representó la mayor proporción de las emisiones totales en toda la serie histórica (86\%), mientras que la Selva Misionera y el Espinal contribuyeron con 5\% cada uno, y la Selva Tucumano Boliviana con sólo el 4\%. El NREF resultó de 101.4 Mt $\mathrm{CO}_{2}$ a ser aplicable durante el período 2014-2018 (Programa Nacional ONU-REDD, 2019).

\section{CONCLUSIONES}

Los bosques nativos del Espinal (Distrito del Ñandubay, Entre Ríos) almacenaban, al año 2007, un total de $54.48 \mathrm{Tg} \mathrm{C}$, con un promedio de 38.82 Mg.ha-1. Estas reservas de $\mathrm{C}$ podrían convertirse en emisiones del orden del 17.7 $\mathrm{Tg} \mathrm{CO}_{2}$ en el período 2020-2030, si las tasas de deforestación se mantienen próximas al 1\% anual. En este contexto, las áreas evaluadas tienen alto potencial para ser incluidas en proyectos REDD+, potenciando la 
conservación del carbono en estos ecosistemas y, con ello, apoyando las metas de reducción de emisiones del país.

Los resultados obtenidos podrían ser útiles como estimados de emisiones ex ante en el planteamiento de proyectos REDD+. Las estimaciones realizadas se refieren sólo a emisiones de $\mathrm{C}$ por deforestación, sin incluir la degradación forestal (aspecto que adolece de vacío de información para bosques en Argentina) ni el reemplazo de las áreas deforestadas por otra cobertura vegetal que capture $\mathrm{CO}_{2}$ atmosférico. En este sentido, este estudio constituye una primera aproximación, siendo prioritario dar continuidad a investigaciones en la temática.

\section{AGRADECIMIENTOS}

Este trabajo fue financiado por el proyecto GEF PNUD ARG/10/G49-PNUMA 4B 85: "Incentivos para la conservación de servicios ecosistémicos de importancia global"; por el PID-UNER 2223: "Captura y fijación de carbono como servicio ambiental de los bosques nativos del Espinal" de la Universidad Nacional de Entre Ríos; y por el Proyecto INTA PE I020: "Desarrollo de criterios para diseño, monitoreo y evaluación de estrategias de intensificación sostenible de agroecosistemas, basadas en múltiples servicios ecosistémicos".

\section{CONFLICTOS DE INTERÉS}

Los autores declaran no tener conflicto de intereses.

\section{CONTRIBUCIÓN POR AUTOR}

M. W., H. A. y S. S. idearon la investigación. E. G., S. S., L. R., S. L. y M. S. Ilevaron la investigación en terreno. S. S., H. A. y M. W. analizaron los datos y redactaron el manuscrito. Todos los autores contribuyeron a la discusión y comentaron las versiones borradores.

\section{REFERENCIAS}

Atencia, M. E. (2003). Densidad de maderas $\left(\mathrm{kg} / \mathrm{m}^{3}\right)$ ordenadas por nombre común. INTI- CITEMA.

Cabrera, A. L. (1976). Regiones fitogeográficas argentinas. Enciclopedia Argentina de Agricultura y Jardinería ( $2^{\mathrm{a}}$ ed.). ACME. S.A.C.I.

Conti, G., Coirini, R. y Zapata, R. (2008). Funciones de Estimación de Volumen de P. nigra var. ragonesei (algarrobo amarillo) en un Bosque del Espinal Santafesino. Ciencia, 3(7), 39-51.

Conti, G., Pérez-Harguindeguy, N., Quetier, F., Gorné, L. D., Jaureguiberry, P., Bertone, G. A., Enrico, L., Cuchietti, A. y Díaz, S. (2014). Large changes in carbon storage under different land-use regimes in subtropical seasonally dry forests of southern South America. Agriculture, Ecosystems \& Environment, 197, 68-76.

http://doi.org/10.1016/j.agee.2014.07.025

FAO. (2018). El estado de los bosques del mundo. Las vías forestales hacia el desarrollo sostenible. http://www.fao.org/3/i9535es/i9535es.pdf

García-Idárraga, F. (2013). Cambio climático y aplicación de proyectos REDD+ en Colombia [Tesis de Maestría, Universidad de Palermo]. Repositorio de la Universidad de Palermo.

https://dspace.palermo.edu/dspace/handle/10226/992

Gasparri, I. y Manghi, F. (2004). Estimación de volumen, biomasa y contenido de carbono de las regiones forestales argentinas (Informe Final). Dirección de Bosques, Secretaría de Ambiente y Desarrollo Sustentable de la Nación.

IPCC. Intergovernmental Panel Climate Change. (2007). Climate Change 2007. Mitigation of Climate Change. Technical Summary.

https://www.ipcc.ch/report/ar4/wg3/

IPCC. Intergovernmental Panel Climate Change. (2020). El cambio climático y la tierra. Informe especial del IPCC sobre el cambio climático, la desertificación, 
la degradación de las tierras, la gestión sostenible de las tierras, la seguridad alimentaria y los flujos de gases de efecto invernadero en los ecosistemas terrestres. OMM-PNUMA.

https://www.ipcc.ch/site/assets/uploads/sites/4/ 2020/06/SRCCL_SPM_es.pdf

MAyDS. Ministerio de Ambiente y Desarrollo Sustentable de la Nación Argentina. (2017). Plan de Acción Nacional de Bosques y Cambio Climático. https://redd.unfccc.int/uploads/4849_1_plan_de_ accion_nacional_de_bosques_y_cambio_climatiCO_-_argentina.pdf [Consulta 23/05/2020].

Muñoz, J., Milera, S., Romero, E. y Brizuela, A. (2005). Bosques nativos y selvas ribereñas en la provincia de Entre Ríos. Insugeo Miscelánea, 14, 169-182.

Newton, P. y Benzeev, R. (2018). The role of zerodeforestation commitments in protecting and enhancing rural livelihoods. Current Opinion in Environmental Sustainability, 32, 126-33. https://doi.org/10.1016/j.cosust.2018.05.023

Pacheco, P., Bakhtary, H., Camargo, M., Donofrio, S., Drigo, I. y Mithöfer, D. (2018). The private sector: can zero deforestation commitments save tropical forests? In A. Angelsen, C. Martius, V. De Sy, A. E. Duchelle, A. M. Larson and T. T. Pham (Eds.), Transforming REDD+: Lessons and new directions (pp. 161-173). CIFOR. https://doi.org/10.17528/cifor/007045

Pardos, J. A. (2010). Los ecosistemas forestales y el secuestro de carbono ante el calentamiento global. Instituto Nacional de Investigación y Tecnología Agraria y Alimentaria Ministerio de Ciencia e Innovación.

http://www.inia.es/gcontrec/pub/60587OT_LIBRO _WEB_1277883079734.pdf

Programa Nacional ONU-REDD. (2019). Nivel de referencia de emisiones forestales de la República Argentina.

https://redd.unfccc.int/files/2019_submission_frel_ argentina.pdf

Sabattini, R. A., Sione, S. M., Ledesma, S. G., Sabattini, J. y Wilson, M. G. (2016). Estimación de la pérdida de superficie de bosques nativos y tasa de deforestación en la Cuenca del Arroyo Estacas (Entre Ríos, Argentina). Revista Científica Agropecuaria, 20 (1-2), 45-56.
Secretaría de Ambiente y Desarrollo Sustentable de la Nación. (2007). Primer Inventario Nacional de Bosques Nativos: informe regional Espinal. Segunda Parte ( $1^{\mathrm{a}}$ ed.).

Sione, S. M., Andrade-Castañeda, H. J., Ledesma, S. G., Rosenberger, L. J., Oszust, J. D. y Wilson, M. G. (2019a). Aerial biomass allometric models for Prosopis affinis Spreng. in native forests of Argentina. Revista Brasileira de Engenharia Agrícola e Ambiental, 23(6), 467-473.

https://doi.org/10.1590/1807-1929/agriambi.v23 n6p467-473

Sione, S. M. J., Ledesma, S. G., Rosenberger, L. J., Oszust, J. D., Carpp, I. A., Wilson, M. G., Andrade Castañeda, H. J. y Sasal, M. C. (2019b). Fracción de carbono en la biomasa de Prosopis affinis Spreng. en un bosque nativo del Espinal (Argentina). Agronomía \& Ambiente. Revista de la Facultad de Agronomía UBA, 39(1), 6-15.

Sione, S. M. J., Ledesma, S. G., Rosenberger, L. J., Oszust, J. D., Andrade, H. J., Maciel, G. O. y WiIson, M. G. (2020a). Modelos alométricos de biomasa aérea para Vachellia caven Mol. Molina en bosques nativos del Espinal (Argentina). Quebracho, 28(1,2), 20-33.

Sione, S. M. J., Ledesma, S. G., Rosenberger, L. J., Oszust, J. D., Andrade Castañeda, H. J., Maciel, G. O., Wilson M. G. y Sasal, M. C. (2020b). Ecuaciones alométricas de biomasa aérea para Prosopis nigra Griseb. Hieron "algarrobo negro" (Fabaceae) en bosques de Entre Ríos (Argentina). Agronomía \& Ambiente Revista de la Facultad de Agronomía UBA, 40(1), 63-76.

UMSEF. Unidad de Manejo del Sistema de Evaluación Forestal. (2007). Monitoreo de Bosque Nativo, Período 1998-2002 y Período 2002-2006 (Datos Preliminares).

https://www.argentina.gob.ar/sites/default/files/2006___monitoreo_bosque_nativo_preliminar.pdf

UNFCCC. (2007). Proceedings of UNCCC 2007. https:// unfccc.int/process-and-meetings/conferences/past -conferences/bali-climate-change-conferencedecember-2007/bali-climate-change-conference -december-2007-0 
Wilson, M. G. (2007). Uso de la tierra en el área de bosques nativos de Entre Ríos, Argentina [Tesis Doctoral]. Universidad de La Coruña.
Winjum, J. K., Dixon, R. K. y Schroeder, P. E. (1993). Forest management and carbon storage: an analysis of 12 key forest nations. Water Air Soil Pollution, 70, 19-38. https://doi.org/10.1007/BF01105000

\section{(c) (i) (2)

